\title{
DINÁMICA POBLACIONAL DE CALIROA CERASI L. (HYMENOPTERA: TENTHREDINIDAE) EN CULTIVOS DE CEREZO (PRUNUS AVIUM L.) DEL VALLE INFERIOR DEL RÍO CHUBUT (REGIÓN PATAGONIA SUR-ARGENTINA)
}

\author{
POPULATION DYNAMIC OF CALIROA CERASI L. (HYMENOPTERA: \\ TENTHREDINIDAE) IN CHERRY (PRUNUS AVIUM L.) ORCHARDS OF LOWER \\ VALLEY OF THE CHUBUT RIVER (SOUTH PATAGONIA REGION-ARGENTINE)
}

\author{
Silvina G. Bado ${ }^{1}$
}

\begin{abstract}
RESUMEN
Caliroa cerasi, la "babosita del peral", ocasiona importantes daños en cultivos de cerezo del Valle Inferior del Río Chubut. El principal objetivo del trabajo fue conocer la biología y dinámica poblacional de la especie en esta región, aspectos básicos para el desarrollo de estrategias de manejo enmarcadas en un Programa de Manejo Integrado de Plagas. Para ello se llevó a cabo un monitoreo en tres predios comerciales y en una parcela experimental del INTA Chubut, durante dos campañas (2005/2006 - 2006/2007), que consistió en el uso de trampas cromatográficas para la captura de adultos, mientras que en el caso de los huevos y las larvas se realizó un conteo directo en hojas. En la segunda campaña, con el fin de ajustar técnicas de muestreo, se tuvieron en cuenta dos estratos de la planta (inferior y medio). Las mayores densidades larvales fueron halladas en una producción orgánica. Las fluctuaciones poblacionales indican que la especie presenta en el VIRCH tres generaciones anuales. Sólo se hallaron diferencias significativas en la cantidad de huevos presentes en los estratos inferior y medio en el predio con sistema de conducción "eje central" en el caso de dos fechas, pero no en el caso de las larvas.
\end{abstract}

Palabras clave: Caliroa cerasi, Prunus avium, fluctuación poblacional, muestreo.

\begin{abstract}
Caliroa cerasi, or "pear slug", causes important damages in cherry orchards of the Lower Valley of the Chubut River. The principal aim of this work was to know their biology and poputation dynamic in this region, being these aspects basic to develop management strategies compatible with a Pest Integrated Management Programme. For this purpose, a scouting in three commercial orchards and in an experimental INTA Chubut plot during two consecutives growing seasons (2005/2006 y 2006/2007) was held. These consisted in the adults capture by chromatographic traps meanwhile in case of eggs and larvae, a direct sampling was done. In order to ajust sampling technics, during the second growing season, two stratum of the plant (lower and medium) were considered. Population density was high in an organic production. Population fluctuations indicate that the species present three generations annually in VIRCH. Significant differences in eggs levels between plant stratums were found only in two dates in an orchard conducted by a "central axis" system, but no in larvae case.
\end{abstract}

Key words: Caliroa cerasi, Prunus avium, population fluctuation, scouting.

\section{INTRODUCCIÓN}

El cultivo de cerezo (Prunus avium L.) constituye actualmente una de las alternativas productivas más importantes que se llevan a cabo en la Región Patagonia Sur, siendo su principal destino la exportación a países europeos. La superficie plantada es de aproximadamente 600 hectáreas, las que se encuentran distribuidas en las siguientes áreas: Valle Inferior del Río Chubut (VIRCH) (43ำ16’ LS; 30 m.s.n.m), las proximidades de la ciudad de Esquel ( $42^{\circ}$ 55' LS; 270 m.s.n.m.), Comodoro Rivadavia ( $45^{\circ} 52^{\prime}$ LS; 50 m.s.n.m.) y Sarmiento $\left(45^{\circ} 35^{\prime}\right.$ LS; 270 m.s.n.m.), en la provincia de Chubut, y el Valle

1 Profesional en Protección Vegetal, Grupo Fruticultura-INTA EEA Chubut, Ex ruta 25 km 1480, Trelew Chubut-Argentina, sbado@chubut.inta.gov.ar 
de Los Antiguos (46 32'LS; 30 m.s.n.m.), en la provincia de Santa Cruz (Figura 1) (Sanz, 2005).

La región presenta clima frío-templado y ventoso con dirección prevalente de los vientos oeste y sudoeste, siendo la época primavera-estival la de mayor incidencia. La precipitación media anual es $196,1 \mathrm{~mm}$ siendo el régimen irregular, mientras que la temperatura media anual, $12,7^{\circ} \mathrm{C}$ (máxima media 20,3 y mínima media, $6,1^{\circ} \mathrm{C}$ ) y la humedad relativa promedio 58\% (Arbunies de Mac Karthy, 1994).

Debido a la creciente exigencia en seguridad e inocuidad de los productos por parte de los mercados compradores, a través de la certificación por buenas prácticas agrícolas, se torna necesaria la implementación de un programa de manejo integrado de adversidades fitosanitarias en el cultivo que garantice un producto de calidad y sin presencia de residuos tóxicos.

Las condiciones ambientales de la región no proporcionan graves problemas fitosanitarios, siendo por ello escasos los tratamientos químicos que el cultivo recibe. Sin embargo, Caliroa cerasi (Hymenoptera: Tenthredinidae), vulgarmente conocida como "babosita del peral", es un insecto que puede ocasionar anualmente importantes daños indirectos en las áreas mencionadas. Sus niveles poblacionales son disminuidos únicamente por medio de insecticidas, los que son aplicados en momentos próximos a cosecha (Bado, 2007).

Con el fin de evitar los problemas de residuos en frutos que esto podría acarrear, resulta de interés contar con medidas de manejo alternativas cuidadosas del medio ambiente así como métodos adecuados de monitoreo. Los estudios bioecológicos de la especie resultan indispensables para el desarrollo de estos aspectos. Es por ello que el objetivo del trabajo fue conocer la biología de $C$. cerasi en el Valle Inferior del Río Chubut y su dinámica poblacional, determinando los niveles poblacionales que puede alcanzar, su fluctuación y la distribución de los estados inmaduros (huevos y larvas) sobre las plantas.

La especie es originaria de la región Paleártica y posee una amplia distribución mundial. En Sudamérica está presente en Colombia, Uruguay, Brasil, Chile y Argentina (web5.silverplatter. com/webspirs/show/). Si bien afecta a numerosas Rosáceas, entre las que se encuentran frutales de los géneros Cydonia, Prunus, Pyrus, Rubus, etc., en ensayos de preferencia alimentaria realizados con varias especies: $P$. avium (cerezo), $P$. cerasus (guindo), P. domestica (ciruelo), Prunus amygdalus (almendro) y Pyrus communis (peral), las larvas consumieron un mayor porcentaje de área foliar de discos de cerezo y guindo (Rocca et al., 2005).

Las larvas se alimentan de la cara superior y del mesófilo de las hojas respetando las nervaduras y la epidermis inferior (Figura 2). Las hojas quedan esqueletizadas, toman coloración amarillenta y dan al árbol un aspecto de quemado (Pino et al., 2007) (Figuras 3 y 4). Al disminuir el área fotosintética puede verse afectada tanto la producción del año como la del siguiente. En este caso, debido a la menor capacidad de acumulación de reservas de la planta, quedando de ese modo susceptible al efecto de las heladas y otras adversidades. Sin embargo, aún no se han llevado a cabo estudios que determinen precisamente la incidencia del daño en el rendimiento del cultivo de cerezo.

Carl (1972) menciona que la especie cumple dos a tres generaciones por año en el hemisferio norte atravesando el invierno en estado de prepupa y afirma que la segunda generación es más sincronizada y destructiva que la primera. Los huevos son hallados simultáneamente al período de emergencia de adultos, debido a que las hembras no necesitan copular para oviponer. Dependiendo de la temperatura, el período embrionario puede tener una duración entre 9 a 16 días.

El período larval en condiciones de campo tuvo una duración de dos a tres semanas en noreste de EE.UU. (www.pan.ci.seattle.was.us) y cuatro en Canadá (www.entomogy.umn.edu/cues/IPM-trees/ P. Hm).

En Nueva Zelanda, mediante el uso de trampas pegajosas amarillas, se detectaron dos picos máximos de vuelo: el primero en diciembre y el segundo a fines de febrero/marzo. La máxima densidad larval ocurrió dos semanas posteriores al momento de máxima emergencia de adultos. Las hembras prefieren para oviponer áreas soleadas y reparadas del viento (Van Epenhuijsen \& De Silva, 1991).

En la X Región de Chile ( $40^{\circ}$ latitud sur), los adultos comienzan a emerger hacia fines de octubre, principios de noviembre, lo que se anticipa en regiones ubicadas hacia el norte. Una segunda generación, de muy escasa densidad, ocurre en un corto período de tiempo (enero-febrero). La mayoría de las larvas provenientes de la generación invernante y el total de las de la generación de verano entraron en diapausa, trascurriendo el invierno en estado de 
prepupa. Un cierto número de individuos tendría la capacidad de permanecer en estado de prepupa más de una temporada. Durante todo el ciclo del cultivo se registró la presencia de oviposiciones (Raddatz Rosenberg, 2004). En la zona sur de Chile, el desarrollo larvario puede transcurrir en 21 a 28 días y el pupal en 15 días (Aguilera, 1989).

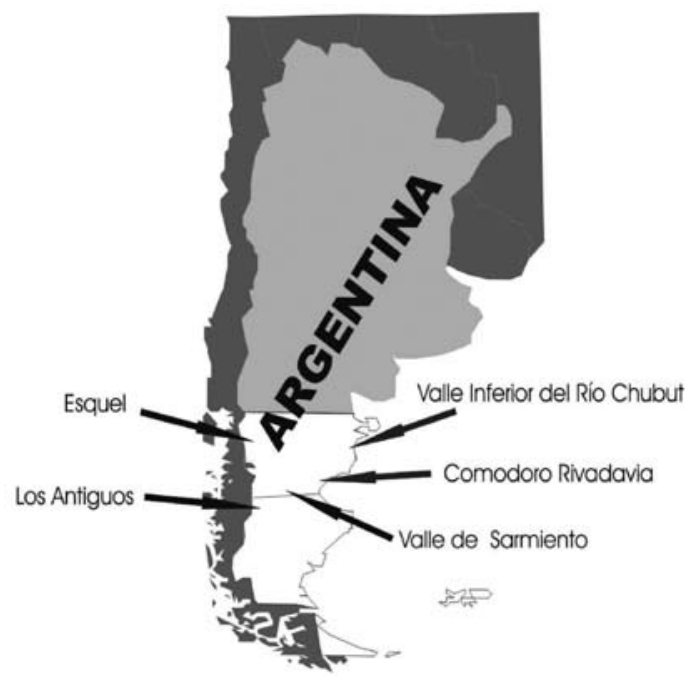

Figura 1. Areas productoras del cultivo de cerezos en la Región Patagonia Sur.

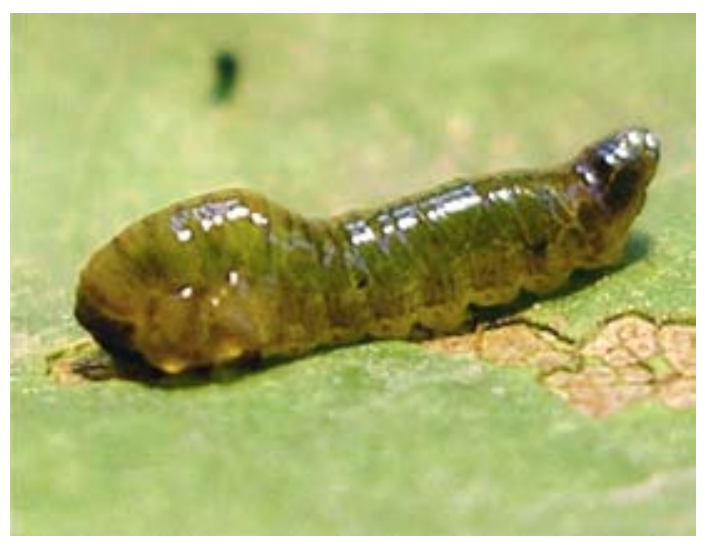

Figura 2. Larva de último estadio de C. cerasi sobre cerezo (Fotografía: S. G. Bado).

\section{MATERIALES Y MÉTODOS}

Los estudios se llevaron a cabo durante las campañas 2005/2006 y 2006/2007 en tres cultivos comerciales ubicados en el Valle Inferior del Río Chubut con distinto manejo de adversidades fitosanitarias (predios 1, 2 y 4) y en una parcela experimental ubicada en la Estación INTA Chubut (predio 3).

El predio 1 corresponde a un cultivo orgánico establecido en el año 2003, conducido mediante el sistema "tatura". Las variedades son Bing, Sweet Heart y Lapins, y el portainjertos, Mahaleb. Se halla protegido de los vientos por medio de cortinas de álamos (Populus sp.) de $25 \mathrm{~m}$ de altura ubicadas al Norte, Sur y Oeste.

En el predio 2 se realizan aplicaciones con agroquímicos. El sistema de conducción es por "vaso español" y se halla protegido por cortinas de álamos de $35 \mathrm{~m}$ de altura hacia el Norte, Oeste y Sur. Las variedades son Van, Bing, Burlat, Napolitana, Lapins y el pie, Mahaleb. En el momento en que se llevó a cabo este trabajo las plantas presentaban ocho años.

En la parcela experimental del INTA Chubut (predio 3) las plantas crecen libremente, presentando distintas edades (7, 2 y 1 año en el momento de la realización del trabajo). Algunas de las variedades son de origen francés mientras que también hay Lapins y Brooks. Al O está protegido por una cortina de "tamariscos" (Tamarix gallica) de $3 \mathrm{~m}$ y hacia el Sur se encuentra una de álamos a $20 \mathrm{~m}$ de distancia del cultivo. Durante el año en que se realizó este trabajo no se aplicaron insecticidas.

El predio 4 fue plantado en el año 1999, estando las plantas conducidas por medio del sistema "eje central". Las variedades son Hedelfingen, Lapins, Stella, Celeste y Kardia sobre pie Mahaleb. Se realizan aplicaciones de agroquímicos. La cortina forestal está conformada por mimbres (Salix sp.), eucaliptos (Eucalyptus sp.), tamariscos y álamos.

Durante la primera campaña el monitoreo se realizó en los predios 1,2 y 3 , mientras que durante la segunda, en los predios 1 y 4 . Para el monitoreo de adultos se colocaron 15 trampas amarillas pegajosas de $15 \times 20 \mathrm{~cm}$, por predio, las que eran ubicadas a una altura del suelo entre 1,5 y $2 \mathrm{~m}$ sobre el tronco de las plantas. La disposición en el predio se hizo siguiendo un recorrido en "zigzag".

En el caso de los huevos y las larvas, en la primera campaña la unidad muestral consistió en 10 hojas tomadas al azar por árbol, seleccionándose por fecha de monitoreo en cada predio 10 árboles al azar, mientras que durante el segundo año el muestreo se realizó en dos estratos de la planta: inferior (desde el suelo hasta un metro de altura) y medio (el metro hasta dos). En cada estrato se tomaron 10 hojas al azar de una rama de $80 \mathrm{~cm}$ aproximadamente. 
Para detectar diferencias significativas entre los niveles poblacionales de huevos y larvas entre estratos se realizó un análisis de ANOVA y Test de Tukey $(\mathrm{P} \leq 0,05)$ por medio del programa Statistics 4.0.

\section{RESULTADOS}

Las Figuras 5, 7 y 8 presentan la fluctuación de los niveles poblacionales de huevos, larvas y adultos durante la campaña 2005/2006, mientras que las Figuras 6 y 9, durante la campaña 2006/2007.

En el predio 1 (Figura 5) los adultos comenzaron a visualizarse en las trampas a mediados de octubre al mismo tiempo que se observaron las primeras oviposiciones en las hojas. La máxima captura de adultos/trampa ocurrió en la primera semana de noviembre. A los 11 días de haberse detectado las oviposiciones, se comenzó a registrar la presencia de larvas. La densidad larval alcanzó un promedio de 5 individuos/ hoja a fines de noviembre ocasionando un importante daño, lo que condujo a realizar una aplicación con Spinosad (5/12) que disminuyó los niveles poblacionales. Posteriormente, se observa la presencia de adultos desde fines de diciembre hasta marzo con un máximo a principios de febrero y de larvas, en menor densidad que en el primer período, desde mediados de enero hasta marzo.

Durante la campaña 2006/2007 (Figura 3) una aplicación de polisulfuro de calcio a comienzos del ciclo del cultivo y dos aplicaciones de Spinosad mantuvieron los niveles poblacionales bajos. Sin embargo, en ambas campañas la fluctuación poblacional resultó similar.

En el predio 2, los adultos y las oviposiciones se detectaron a mediados de noviembre, y al cabo de dos semanas, las larvas (Figura 4). Una aplicación con deltametrina 5\% (CE) a mediados de diciembre disminuyó los niveles poblacionales de esta generación. Desde mediados de enero hasta mediados de marzo la presencia de adultos, huevos y larvas fue continua con excepción de una fecha ( 25 de enero para el caso de los adultos y 1 de febrero para el de larvas), lo que podría estar relacionada a un control químico llevado a cabo con un acaricida (Dicofol 21\% + Tetradifón 7,5\%, $\mathrm{CE})$. Las densidades larvales fueron mayores en este segundo período.

En el predio 3 (Figura 8), los adultos y las oviposiciones fueron detectadas a comienzos de noviembre y las larvas, 15 días más tarde. Durante

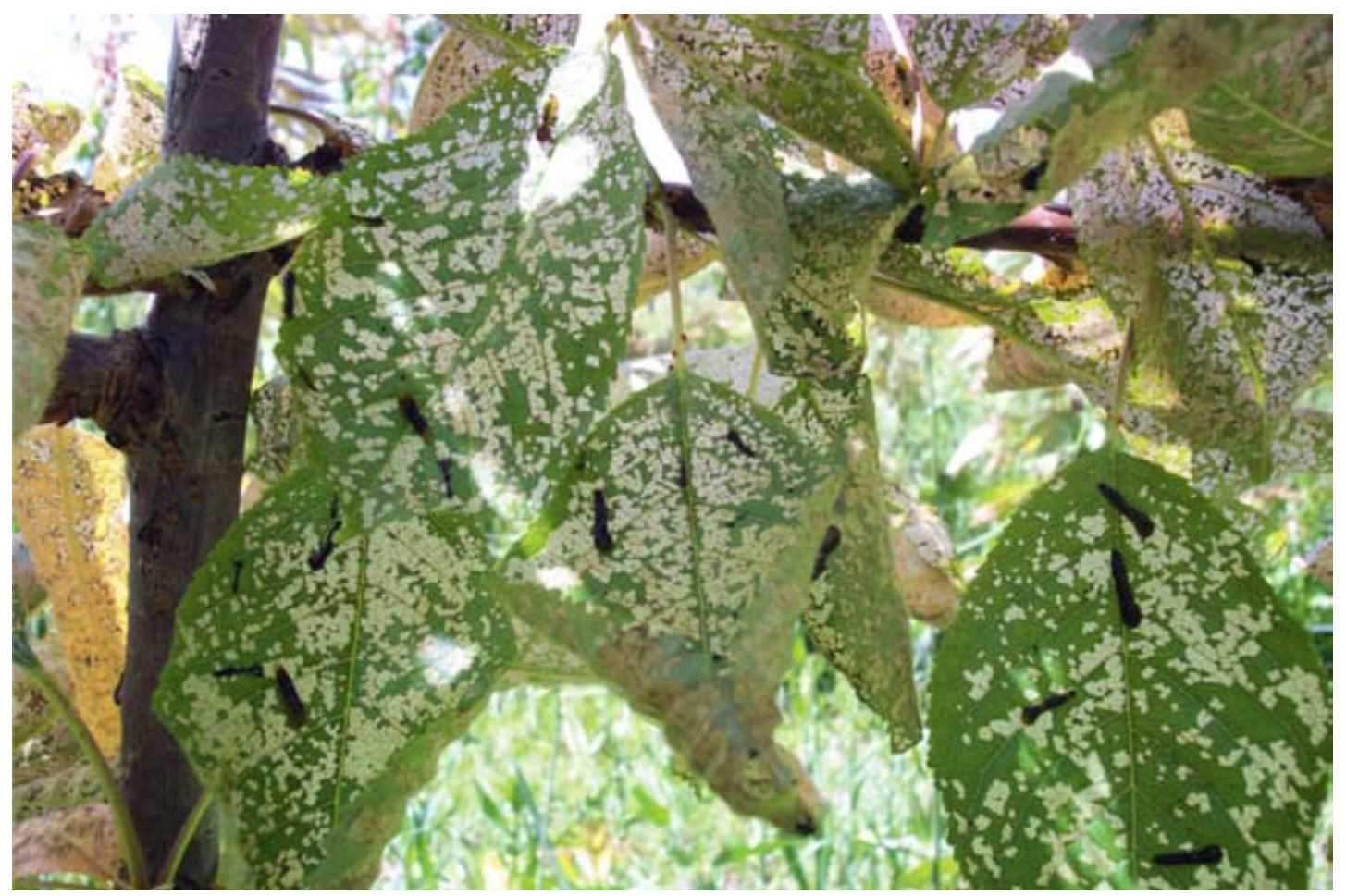

Figura 3. Larvas de C. cerasi y el daño ocasionado en cultivo de cerezos (Fotografía: S. G. Bado). 


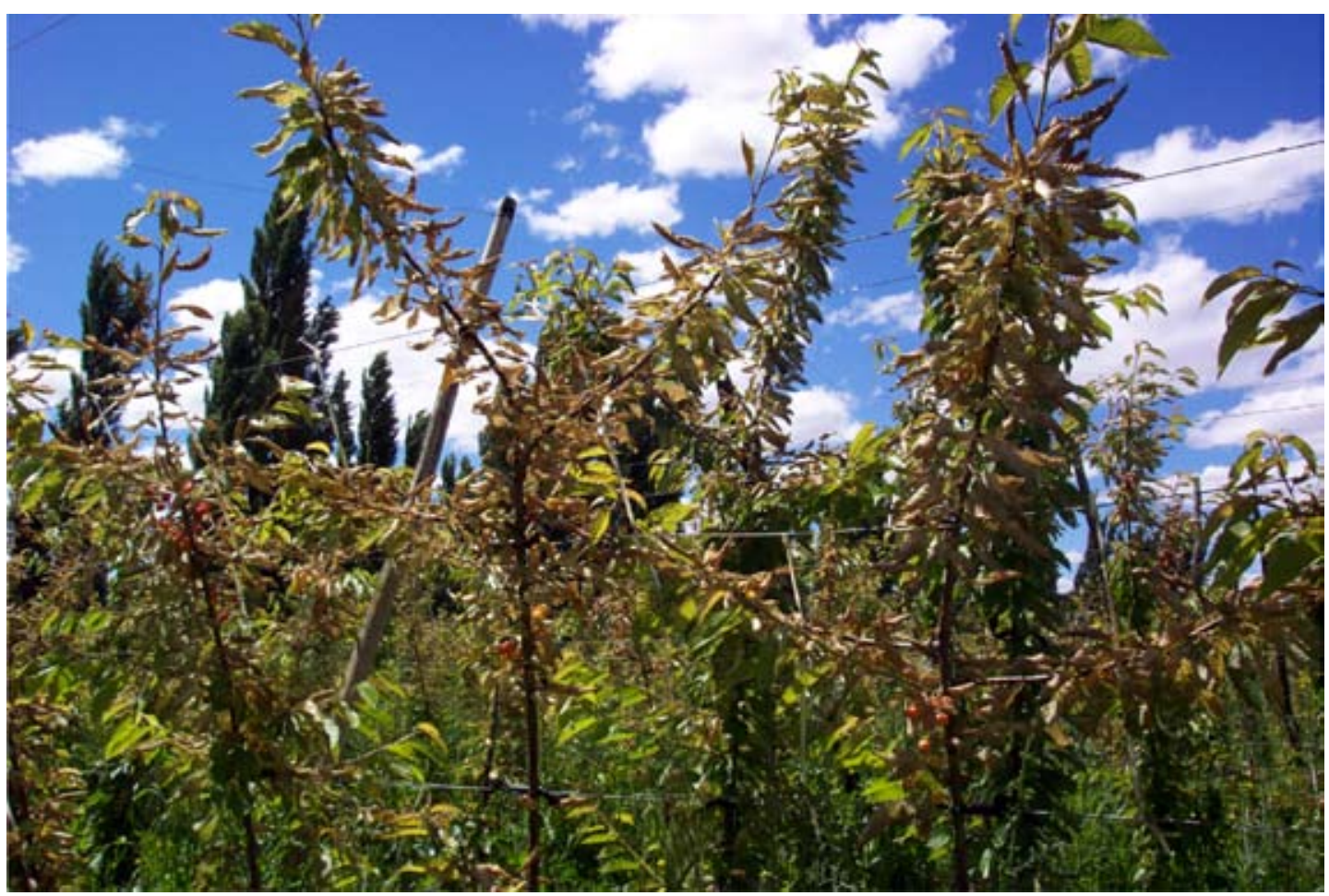

Figura 4: Plantas de cerezo dañadas por C. cerasi.

Fluctuación poblacional de C. cerasi (Hymenoptera: Tenthredinidae) en predio 1 (campaña 2005-2006)

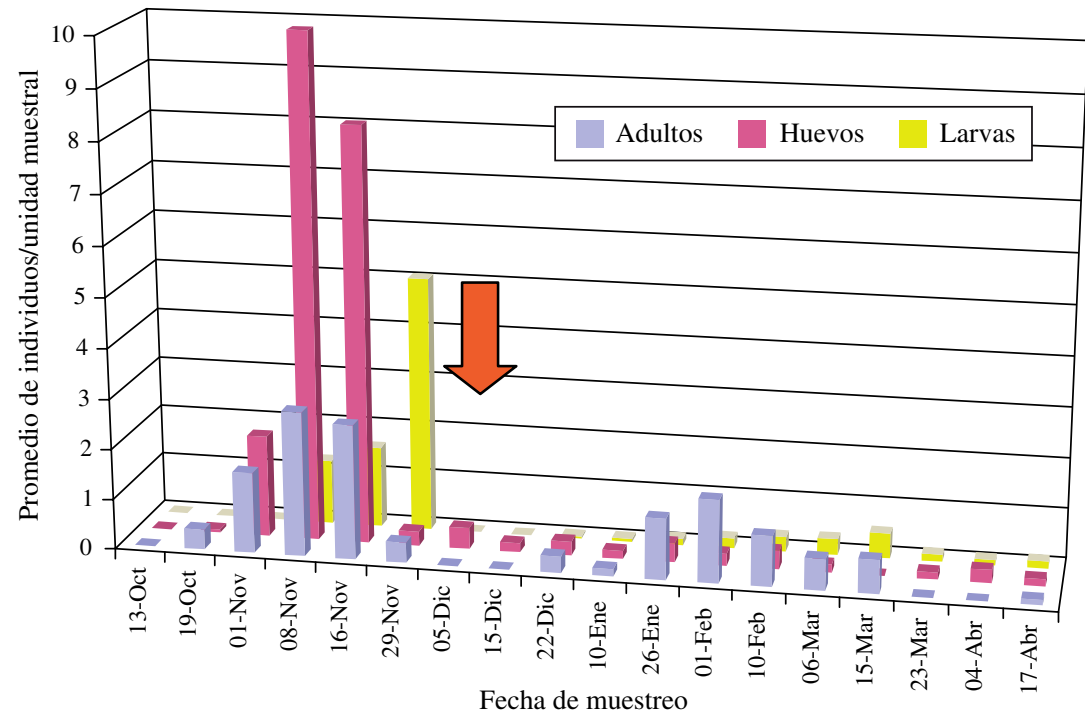

Figura 5: Fluctuación poblacional de huevos, larvas y adultos de $C$. cerasi registrada en el predio 1 durante la campaña 20052006. 
Fluctuación poblacional de C. cerasi (Hymenoptera: Tenthredinidae) en predio 1 (campaña 2006-2007)

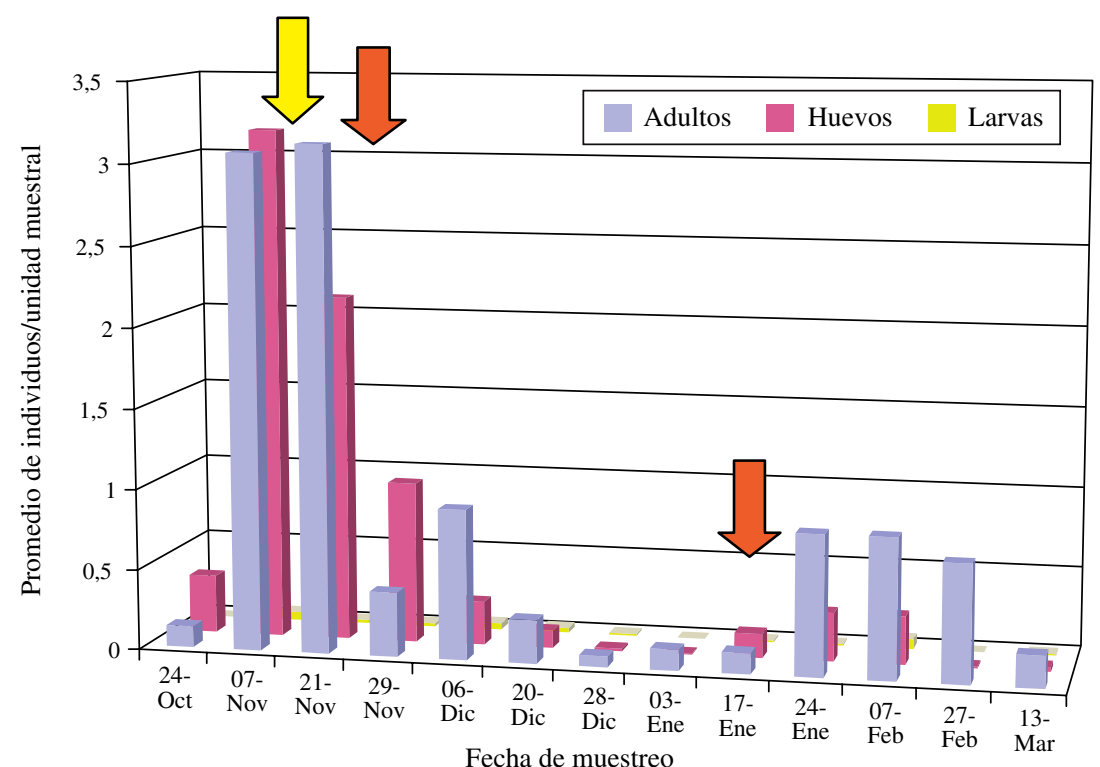

Figura 6: Fluctuación poblacional de huevos, larvas y adultos de C. cerasi registrada en el predio 1 durante la campaña 20062007.

Fluctuación poblacional de C. cerasi (Hymenoptera: Tenthredinidae) en predio 2 (campaña 2005-2006)

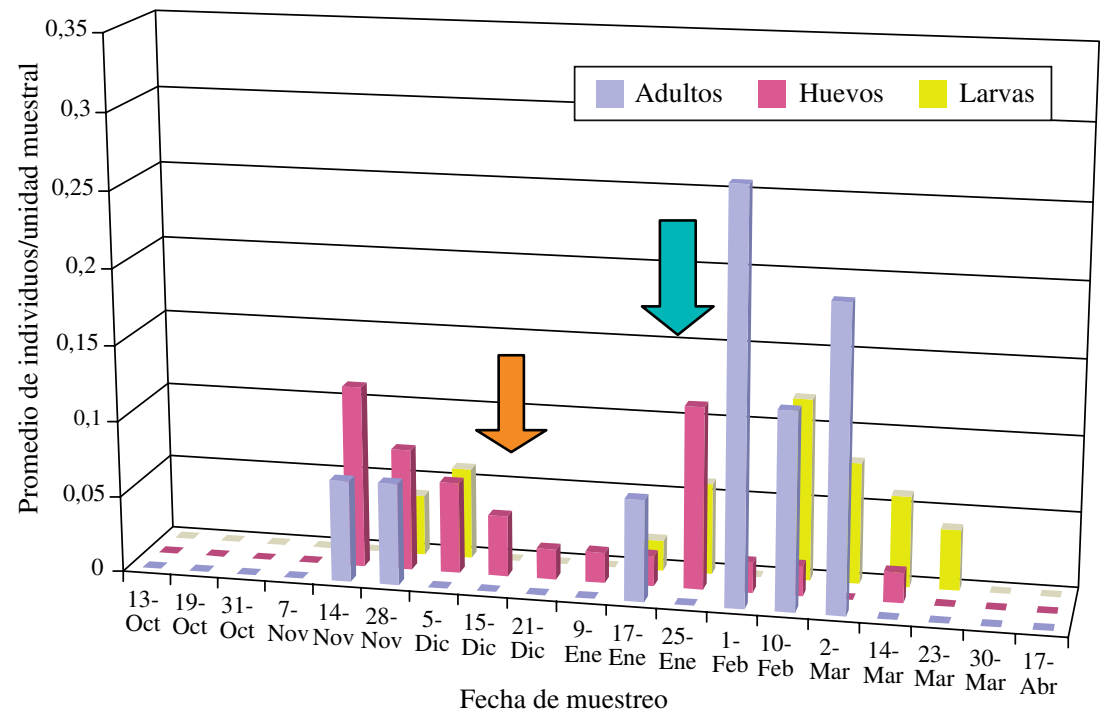

Figura 7: Fluctuación poblacional de adultos, huevos y larvas C. cerasi registrada en el predio 2.

los meses primaverales los adultos sólo fueron detectados en una sola fecha y luego se observan dos picos máximos más: a principios de febrero y mediados de marzo. La máxima densidad larval ocurrió en el mes de febrero.
En el predio 4 (Figura 9), las densidades poblacionales fueron muy bajas y se detectaron a fines de octubre. Nuevamente se registraron dos períodos de ocurrencia: el primero en noviembre donde se presentaron las mayores densidades 
Fluctuación poblacional de C. cerasi (Hymenoptera: Tenthredinidae) en predio 3

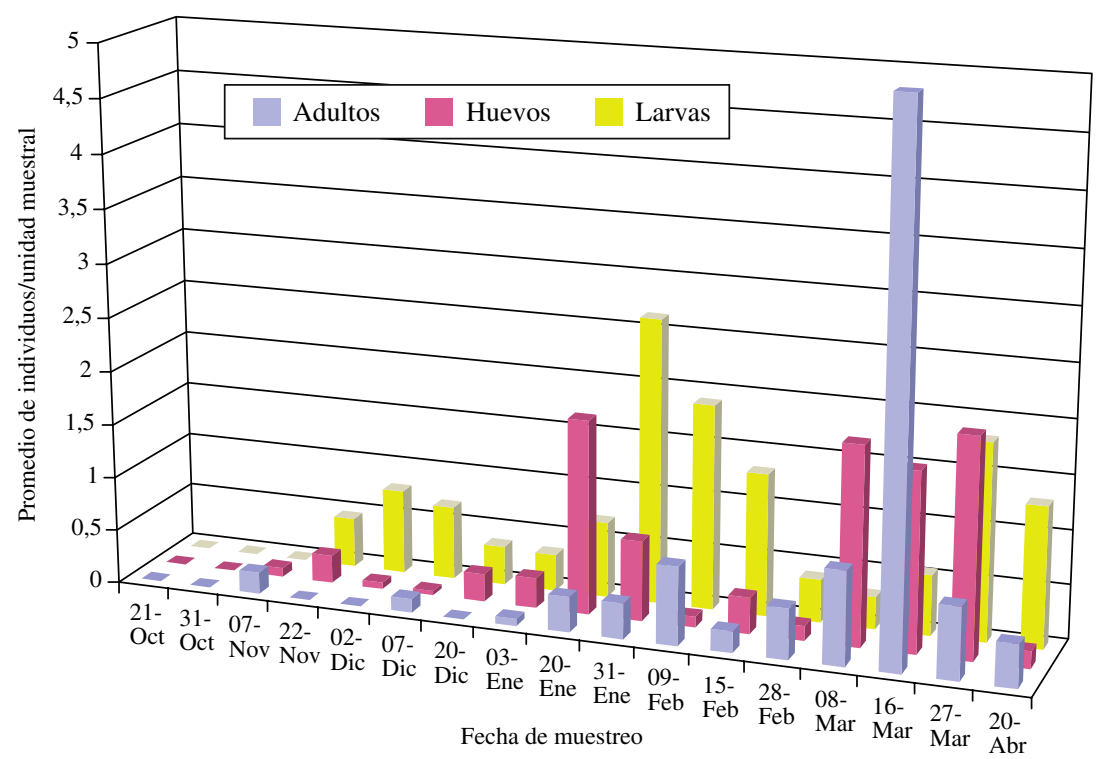

Figura 8: Fluctuación poblacional de adultos, huevos y larvas C. cerasi registrada en el predio 3 (campaña 2005-2006)

Fluctuación poblacional de C. cerasi (Hymenoptera: Tenthredinidae) en predio 4

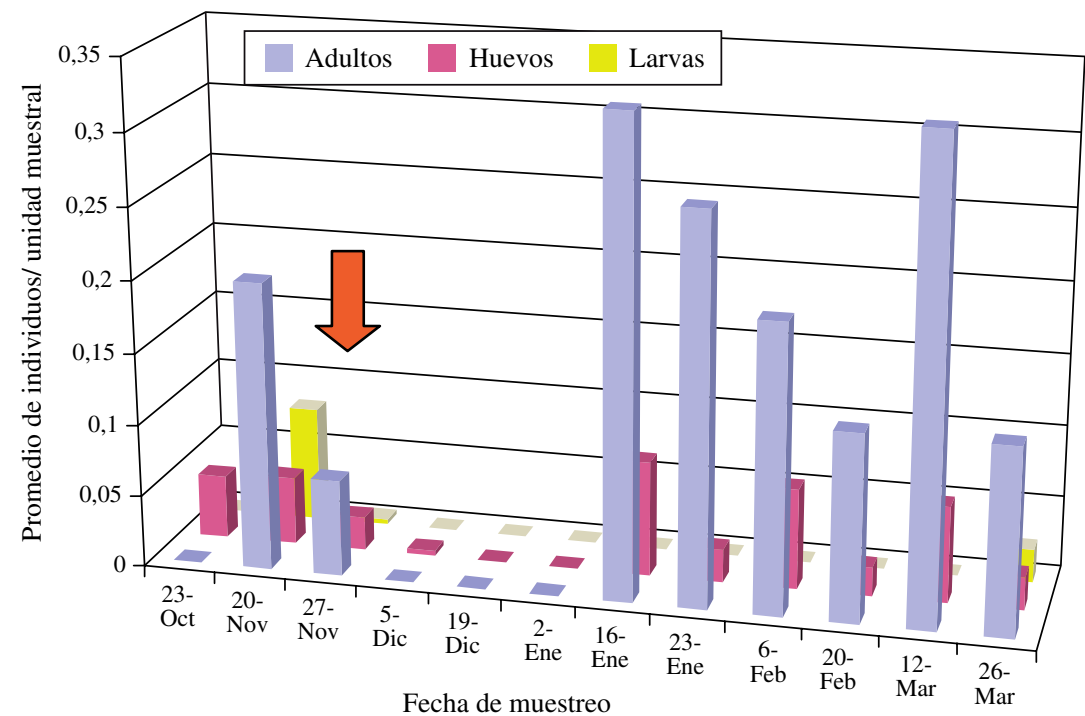

Figura 9: Fluctuación poblacional de adultos, huevos y larvas C. cerasi registrada en el predio 4 (campaña 2006-2007).

larvales que fueron disminuidas por medio de un control químico con lambdacialotrina, y el segundo a partir de mediados de enero hasta marzo donde se registraron adultos principalmente. La presencia de larvas fue escasa ascendiendo recién a fines del mes de marzo.
En las Figuras 10 y 11 se observan los niveles poblacionales de huevos y larvas registrados por estratos en el predio 1, mientras que en las Figuras 12 y 13 , en el predio 4 . La cantidad de huevos fue significativamente mayor $(\mathrm{P} \leq 0,05)$ en el estrato inferior sólo en dos fechas de muestreo (fines de enero y febrero) en el caso del predio 4. 


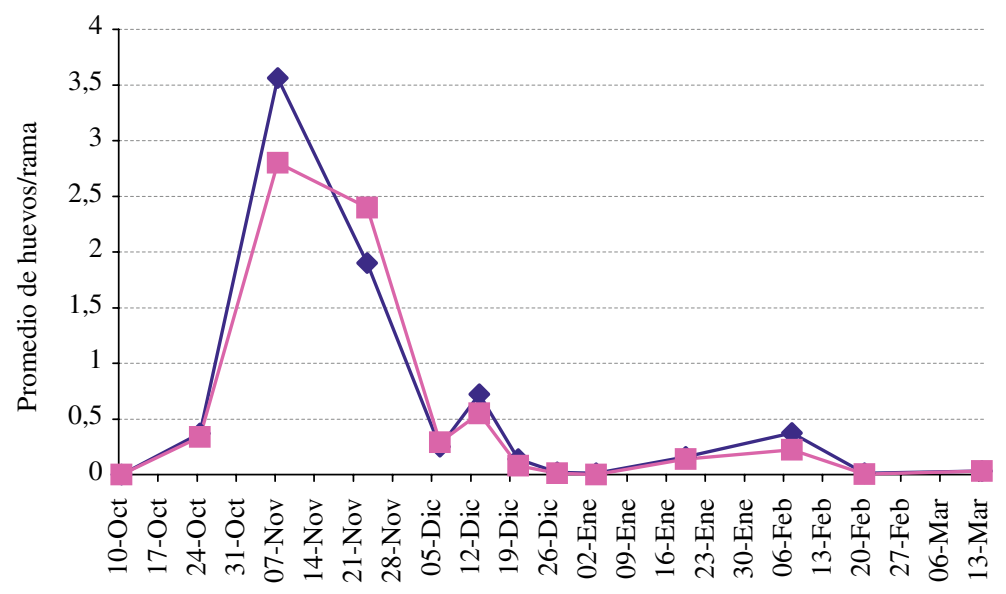

Fecha de muestreo

$\longrightarrow$ Estr. Inf.

Figura 10: Fluctuación poblacional de huevos de C. cerasi en estratos inferior y medio de las plantas en el predio 1.

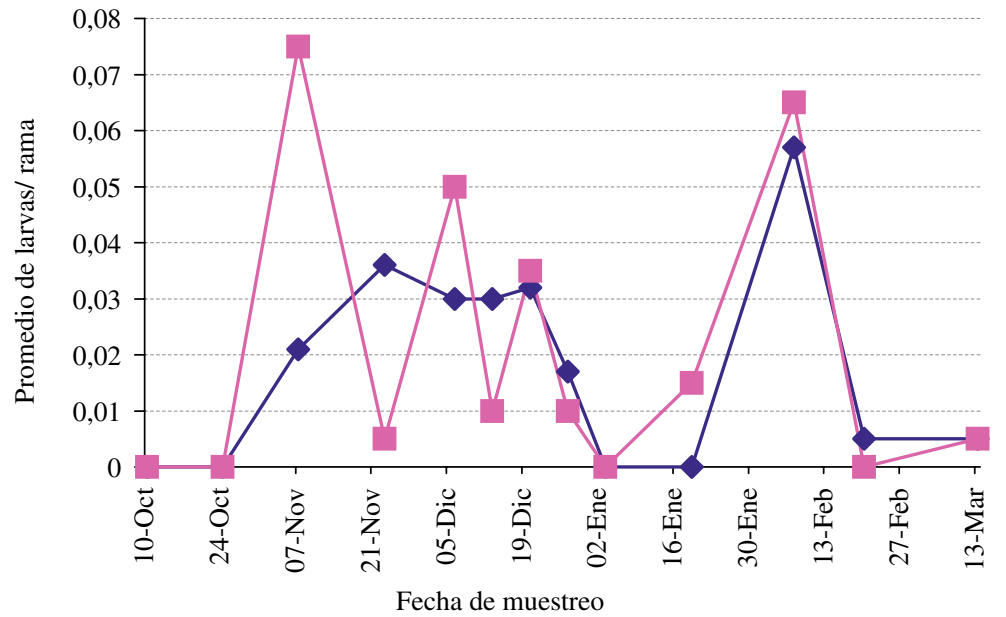

\section{Estr. Inf. $\longrightarrow$ Estr. Medio}

Figura 11: Fluctuación poblacional de larvas de C. cerasi en estratos inferior y medio de las plantas en el predio 1.

\section{DISCUSIÓN}

En el VIRCH, los adultos provenientes de la generación invernante comienzan a emerger desde mediados de octubre y noviembre, según el predio, dando origen a una generación bien definida que transcurre en los meses primaverales. En el caso del predio 3, el registro de adultos sólo en una fecha en esta época, que no se correspondió con la presencia de larvas, podría tener su causa en la mayor exposición del predio a los vientos primaverales que habrían afectado la efectividad de las trampas.
Coincidiendo con lo señalado por Carl (1972), las oviposiciones fueron detectadas junto con la presencia de los adultos, como consecuencia de la reproducción partenogenética que presenta la especie. Por lo general dentro de los 15 días posteriores ocurrió la visualización de las larvas, por lo que el período embrionario comprendería una duración aproximada, tal como lo expuesto por el mismo autor.

En todos los predios los niveles poblacionales volvieron a ascender a fines de enero, principios de febrero persistiendo hasta marzo, dando de ese 


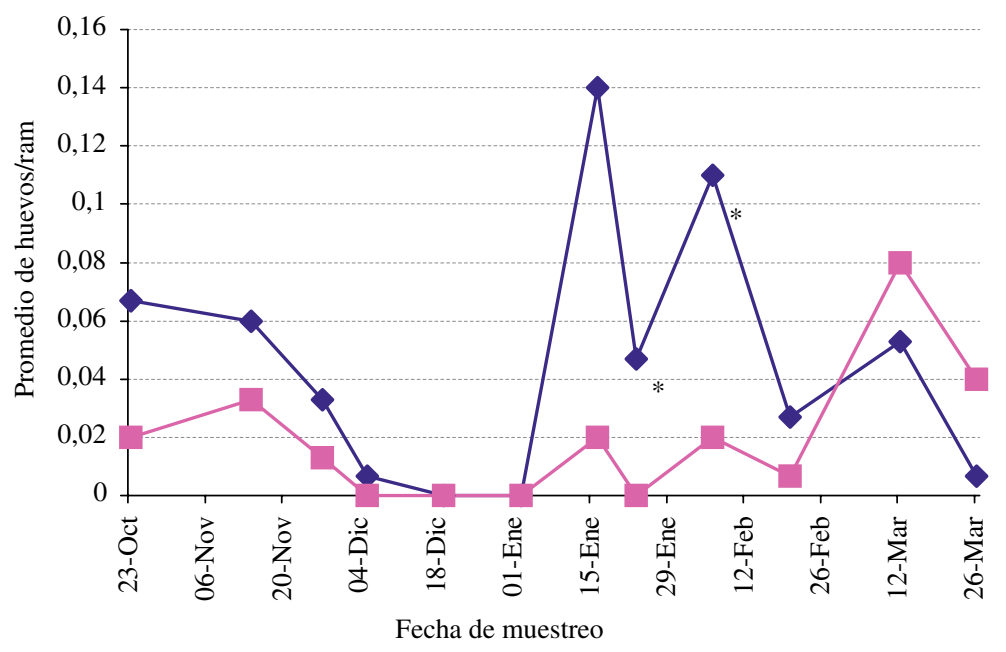

$\longrightarrow$ Estr. Inf. $\longrightarrow$ Estr. Medio

Figura 12: Fluctuación poblacional de huevos de C. cerasi en estratos inferior y medio de las plantas en el predio $4 . *$ indica diferencias significativas $(\mathrm{P} \leq 0,05)$.

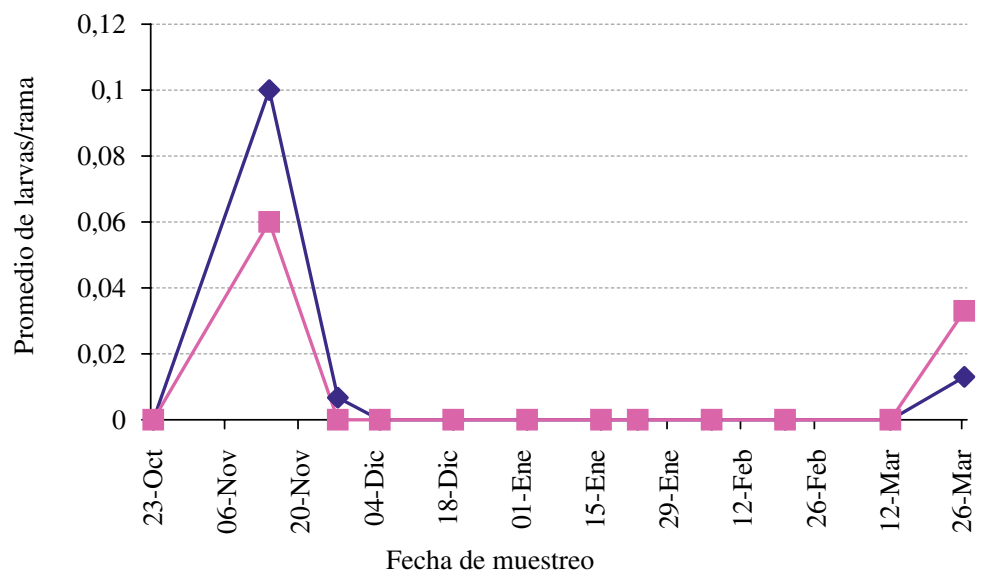

\section{$\longrightarrow$ Estr. Inf. Estr. Medio}

Figura 13: Fluctuación poblacional de larvas de C. cerasi en estratos inferior y medio de las plantas en el predio 4.

modo un amplio período de presencia de la especie difiriendo de ese modo de lo observado en Chile, donde la segunda generación resulta corta y de baja densidad (Raddatz Rosenberg, 2004). Lo hallado coincidiría con lo mencionado por Carl (1972) para el hemisferio norte quien señala de dos a tres generaciones anuales, pero sólo la segunda generación fue bien definida y más destructiva que la primera en el caso de la EEA INTA Chubut.

Las mayores densidades larvales fueron registradas en el predio orgánico durante la primera campaña y en la segunda generación en la parcela experimental INTA Chubut.

Las densidades larvales en el segundo período, en el VIRCH resultaron ser muy variables, lo que denota la importancia de contar con un sistema de monitoreo adecuado para la evaluación de los niveles poblacionales así como la determinación de niveles y umbrales de desarrollo para la especie.

En este trabajo también se registró la presencia de oviposiciones durante todo el ciclo del cultivo, tal como fue señalado por Raddatz Rosenberg (2004). 
Dado que la distribución de la luz solar en el cultivo que se logra con los sistemas de conducción "tatura" y "eje central" es más homogénea con respecto a otros sistemas, las ligeras diferencias halladas en la cantidad de huevos en el caso del predio 4 (eje central) podrían deberse al mayor desarrollo de las ramas que alcanzaron en el estrato inferior, lo que habría ocasionado una menor incidencia del viento, condición favorable para la oviposición tal como lo señalado por Van Epenhuijsen \& De Silva (1991).

\section{CONCLUSIONES}

1 En la región del VIRCH, C. cerasi comienza a registrarse desde mediados de octubre

\section{LITERATURA CITADA}

ARBUNIES DE MAC KARTHY, R. 1994. Estadísticas agro meteorológicas del Valle Inferior del Río Chubut. Publicación del Instituto Nacional de Tecnología Agropecuaria. Centro Regional Patagonia Sur. Estación Experimental Agropecuaria Chubut. 142 pp.

AGUILERA, A. 1989. Ficha entomológica para la IX Región de La Araucanía. Caliroa cerasi (L.) (Hymenoptera: Tenthredinidae). Investigación y Progreso Agropecuario, Carillanca (Chile) 8 (4): s. p.

BADO, S.G. 2007. Plagas del cultivo de cerezo. Revista Fruticultura Profesional $N^{\circ}$ 171. pp. 14: 22.

CARL, K.P. 1972. On the biology, ecology and population dynamic of Caliroa cerasi (L.) (Hym., Tenthredinidae). Zeitschrift fur Angewandte Entomologie (Alemania) 71: 58:83.

PINO, C.; SILVA, G.; HEPP, R.; VENEGAS, F. 2007. Eficacia de Peak Plus en el control de Caliroa cerasi ((Hymenoptera: Tenthredinidae). Cien. Inv. Agr. 34 (1): 23-29.

ROCCA, M.; GRECO, N.; MAREGGIANI, G. 2005. Consumo diferencial de especies de la familia Rosaceae por C. cerasi hasta mediados de noviembre, presentándose una generación primaveral bien definida y posteriormente un largo período de presencia que correspondería con dos generaciones más.

2 En el sistema de conducción tipo "eje central" y "tatura" no se hallaron diferencias significativas en la densidad de larvas entre los estratos inferior y medio de las plantas y sólo escasas diferencias en el caso de las oviposiciones.

\section{AGRADECIMIENTOS}

A Nelson Hughes por su colaboración en la toma de datos.

(Hymenoptera: Tenthredinidae). Resúm. VI Congreso de Entomología, Tucumán, 12 y 15 de septiembre.

RADDATZ ROSENBERG, L. 2004. Emergencia de la generación invernante y diapausa de Caliroa cerasi (L., 1758) bajo condiciones de campo en Valdivia, Décima Región de Los Lagos. Universidad Austral de Chile. Facultad de Ciencias Agrarias Escuela de Agronomía. 94 pp.

SANZ, C. 2005. Relevamiento del sector cerecero de Valle Inferior del Río Chubut. Informe técnico, INTA-EEA Chubut, Argentina. 8 pp.

VAN EPENHUIJSEN, C.W.; DE SILVA, H.N. 1991. Monitoring and control of pear slug (sawfly) in an organic nashi orchard. Proc. 44 th N. Z Weed and Pest Control Conf. 80-85.

web5.silverplatter.com/webspirs/show/. Caliroa cerasi. 11/07/2006.

www.pan.ci.seatle.wa.us -Pear slug (Caliroa cerasi) 22/04/2005.

www.entomogy.umn.edu/cues/IPM-trees/ P. Hm IPM Manual of tree Insects 13/04/2005. 